\title{
Come Along: understanding and motivating participation to social leisure activities
}

\author{
Beatrice Valeri, Marcos Baez, Fabio Casati \\ Department of Information Engineering and Computer Science \\ University of Trento, Italy \\ Via Sommarive, 5 I-38123 Trento \\ \{valeri, baez, casati\}@disi.unitn.it
}

\begin{abstract}
In this paper we study the factors that affect people's decision in participating in leisure activities in the social and cultural environment. To this end, we collected the ratings of local people from three different cities around the world on standard leisure activities, and looked at the personal, social and contextual features shaping their preferences. We then used this dataset to evaluate how these features can be exploited to recommend places people would actually like. Our initial results suggest that friends are a good source for recommending places, with higher precision and recall than considering only popular places; but these can be improved reducing the scope to similar friends in the context of the particular activity. We have also found that people preferences are sensitive to the companion (e.g., partner, friends, tourists) for which they look for different features. The results also suggest that similarities in the preferences of people can be extended to other activities, which points to the potential of profiling users based on lifestyle. We finally present the design and prototype of a system, namely ComeAlong, which aims at helping people discover, find and participate to social and leisure activities.
\end{abstract}

Index Terms-Social persuasion, intention sharing, collaborative filtering

\section{INTRODUCTION}

In recent years, we have seen a growing number of competing options trying to get a slice of our leisure time. Having options in principle is positive, but when the number start affecting our ability to choose what activity to perform and where, then we end up with the feeling that we are missing out more interesting options. A complementary problem can be seen from the perspective of someone organising an activity: there are so many options that it becomes difficult to reach those who might be interested.

The problem of finding interesting activities to perform in leisure time is well known and there are several online services such as TripAdvisor ${ }^{1}$, Foursquare $^{2}$, Yelp $^{3}$ and Evenbrite ${ }^{4}$ providing different types of suggestions for events or places. While these services do focus people's attention to a reduced number of events, in most cases people still have the feeling of missing out interesting activities [1]. This calls for a better understanding of the dynamics around people's decisions to participate of activities.

\footnotetext{
${ }^{1}$ http://tripadvisor.com/

${ }^{2}$ https://foursquare.com

${ }^{3} \mathrm{http} / / / \mathrm{www}$.yelp.com

${ }^{4} \mathrm{http}: / / \mathrm{www}$. eventbrite.com
}

In this paper we study how people decide what activity to perform during leisure time, and in particular in which places they can perform the chosen activity, focusing our attention to typical activities that are performed on a standard evening in three different cities in the world: drinking aperitivo in a bar and having dinner at a restaurant in Trento, Italy; having dinner at a restaurant and drinking some beer in a pub in Asunción, Paraguay; having dinner at a restaurant and dancing in a club in Tomsk, Russia. We discovered that places for performing these activities are chosen differently according to the kind of companions people are spending their leisure time with, and in which situations price / quality ratio is important.

Recommendations are analysed too, first searching which user-base makes collaborative filtering more effective, then finding that recommendations can be computed also across different activities: knowing user's tastes about restaurants, we can recommend which places she can like for the other activity considered in her city. This last discovery leads to the possibility of profiling users based on their lifestyle, allowing us to extend the recommendation service to all different kinds of leisure activities maintaining the high quality.

As important as helping people find interesting activities, we also focus on the complementary problem of how activity organisers could motivate people to participate of social activities. We move our focus to an environment where the impact of our ideas can be computed more easily: museum exhibitions and events. This context is particularly interesting because museums, being public services, have less possibility to increase their visibility with expensive advertisement and can receive high benefits from a better usage of free advertisement through the Internet. Our study on the museum environment shows that word of mouth is an important trigger for the decision to go to the museum, but it can be improved by increasing its diffusion through social networks.

All the findings coming from the studies presented in this paper are considered in the design of the ComeAlong platform, which i) supports the discovery of events and activities, and ii) motivates people to participate exploiting the power of online word of mouth.

The main contributions of this paper can be summarised as:

- a large scale study on three different cities around the world, gathering a total of 9820 ratings from 162 local people. 
- an analysis of factors affecting people's decision and preferences in participating in leisure activities, considering the social context, preferences across activities and the effect of individual's goals in engaging in an activity.

- a preliminary analysis in the context of cultural activities aiming at identifying how museums can attract more people to their events, resulting in the design of a supporting tool namely ComeAlong for museums.

In the following we detail on the related work, studies and design of the ComeAlong tool.

\section{RELATED WORK}

The works related to the topic of this paper comes from two main areas: services that support the discovery of leisure events and activities, and recommendations. The first part is about gathering the information about events and show it to users, making them aware of all their possibilities. Then, recommendations help users to find what is interesting for them out of the large amount of information.

\section{A. Search and discovery}

The Internet is a perfect place where people can discover and advertise events and activities. When you want to search which events are available in your city, you can check them on Zvents ${ }^{5}$, Yelp ${ }^{6}$, Upcoming by Yahoo $^{7}$ or Eventbrite ${ }^{8}$, just to name some of the most famous services. They collect information about popular events and provide users support for searching what they are interested in mainly through filters by dates, location, category or price range. These websites are good because they collect in one place a big amount of information about social events, but their lists usually are not complete and their accuracy depends on the location where the user lives.

An important characteristic that these services do not take into consideration is the social aspect of events. When people decide whether to attend or not an event, they take in consideration also who of their friends is going to attend it and sometimes this information make them participate even if it is farther than the distance they usually cover for attending events [2]. Social websites are a good place where the social information can be linked to events, like in Facebook events ${ }^{9}$ and Google+ events ${ }^{10}$.

There are also other services that recommend places where people can spend their leisure time. TripAdvisor and other recommendation systems based on users' feedback have a widely recognized importance in people's choice of which hotel to book or in which restaurant to go for dinner ([3], [4]). The ratings present in such websites are considered even of better quality than the ones given by experts, since experts can give biased opinions for personal profit. The problem

\footnotetext{
${ }^{5}$ http://www.zvents.com

${ }^{6}$ http://www.yelp.com

${ }^{7} \mathrm{http}$ ://upcoming.yahoo.com

${ }^{8}$ http://www.eventbrite.com/

${ }^{9}$ https://www.facebook.com/events

${ }^{10} \mathrm{https} / / /$ plus.google.com/events
}

of false reviews given by the owners of the represented businesses do not make the system unreliable thanks to the big number of honest reviews that make them inefficient [5]. Users' satisfaction of TripAdvisor recommendations has not been studied yet.

Location-based applications are also used by many people to decide where to go when they have free time. It allows them to know where their friends are in a specific moment and meet new people that are in the same place. Foursquare is one of the most famous and is based on manual check-ins to associate users' position to a specific venue. Location is shared for different reasons that go from life-logging, to communication and coordination with companions, to collection of rewards [6]. Self-representation, instead, is one of the mayor reasons why people do not check-in: if people go to a fast food while all their friends consider important to eat well, they do not check in because they do not want their friends to know that they eat bad-quality food [7]. Foursquare is widely used for discovering new places and have serendipitous faceto-face meetings with friends, but it does not support events or planning of meetings with friends.

There are studies about the behaviour of people through their location sharing on social networks and designed some models to predict their future checkins ([8], [9], [10]). Chang and Sun [9] found that people's location is clearly influenced by their friends' ones: knowing the position of friends and the previous checkins, they can predict the next checkins with $90 \%$ of precision. This is an important hint on how to understand people's tastes if we know their previous locations, while in this paper we are going to study how to suggest interesting activities and places people don't know yet.

\section{B. Recommendations}

Recommendations have been deeply studied in the last years. Collaborative filtering was quite famous, but in 2001 it was already clear that it was not enough [11]. Collaborative filtering builds recommendations using other users' opinions of the items: it uses people's ratings to predict how much the user would like an item. Mui and his colleagues already understood that each person gives different reputation to others and their opinions should have different effects on the predicted rating of the user computed by the collaborative filtering algorithm. Other researchers used profile similarity and rating overlap to improve the quality of recommendations [12]. Since offline recommendations are received mainly from friends and familiar advisors, i.e. people that the user knows and trusts, Bonhard and his colleagues proposed to apply collaborative filtering only on a more personal user-base: people that have the same interests (coming from their profiles) or that rated the same items in the same way.

In the last years, thanks to social networks, more people's information became available on the Internet: friendship and trust relationships. Such information can be used to improve the recommendations generated with collaborative filtering ([13], [14], [15]). In [13] the authors found that there are two types of relations that, combined together, enhance the quality 
of recommendations: they called them social friendship and spiritual friendship. Social friendship is the actual friendship relation stated by users, while the spiritual relation is defined by the similarity in behaviour, i.e. two users have a spiritual friendship when they like the same things. The conclusions in [14] are quite similar: when collaborative filtering is applied on a user-base composed of user's friends and neighbours (i.e. people with similar tastes) the resulting recommendations are better than using only one of the groups. Going more deep in the recommendation of events, in [16] the authors clarified the strong relation between events and friends participating to it, building a recommender system for events that give priority to the ones that are going to be attended by user's friends.

But collaborative filtering has two known problems: cold start and sparsity. They can be solved or at least mitigated with a cross-domain approach [17]. Cross-domain collaborative filtering allows recommendation systems to consider the information collected in one domain to improve recommendations for a different domain. This approach has been tested in domains like movies, TV shows and books, where only tastes are concerned. In this paper, we will focus on recommendations for places where people can perform leisure activities, paying attention to the strong social component of this specific context, which is very important as we have seen before.

\section{UNDERSTANDING USER INTENTION AND PREFERENCES}

In order to gain a better understanding of the factors shaping user preferences in participating to leisure activities, we run two separate experiments, each looking at different properties of the user and activities.

In the first experiment, we collected ratings for the places that provide one of the most common activities performed in leisure time: restaurants. We focused on the top 75 restaurants in Trento, Italy, and we built a website where citizens could access using their Facebook account and could give a rating to the places they know. Together with the ratings, we collected also the friendship relations between users (i.e. which users are also friends on Facebook), in order to study whether friends' opinions can provide better recommendations than general users and users with similar tastes. The results of this study have been already published in [1] and will be shortly recalled later.

In the second experiment, we extended the first one in three directions: i) we run it in 3 different cities around the world (Trento in Italy, Asunción in Paraguay, and Tomsk in Russia); ii) we considered two different leisure activities in each city, one of which is again restaurants while the other is different for each city; iii) for each place, users were able to specify 4 different ratings according to different goals that can be accomplished with an activity.

In the following, we describe the methodology and report on the results.

\section{A. Formal definitions}

Before describing the studies in details, we start by providing some formal definitions.

Let $\mathcal{U}$ be the set of all users and $\mathcal{P}$ the set of all the places where activities can be performed. Let $\mathcal{A}$ be the set of all activities, and $\mathcal{G}$ the set of goals that can be accomplished with an activity. Liked represents the relation $(u, p, a, g) \in \mathcal{U} \times \mathcal{P} \times$ $\mathcal{A} \times \mathcal{G}$ of places users rated positively for a given activity on a specific goal, and Disliked the relation $(u, p, a, g) \in \mathcal{U} \times \mathcal{P} \times$ $\mathcal{A} \times \mathcal{G} \backslash$ Liked of places users rated negatively. As an example, this definition can capture a situation in which a user $(u)$ likes a given restaurant $(p)$ to have dinner $(a)$ with her partner $(g)$.

Given these basic elements we define:

$$
\begin{aligned}
\operatorname{Rated}(u, p, a, g) & =\operatorname{Liked}(u, p, a, g) \vee \operatorname{Disliked}(u, p, a, g) \\
\operatorname{Known}(u, a) & =\{p \in P \mid \exists g \in G, \operatorname{Rated}(u, p, a, g)\} \\
\text { Unknown }(u, a) & =\mathcal{P} \backslash \operatorname{Known}(u, a)
\end{aligned}
$$

In the studies we considered two particular relations between users: friendship and similarity. FriendOf denotes the relation $(u, u) \in \mathcal{P}^{2}$ and represents the symmetric friendship relation used in Facebook:

Friend $\mathrm{Of}\left(u, u^{\prime}\right) \Leftrightarrow$ FriendOf $\left(u^{\prime}, u\right) \Leftrightarrow \mathrm{u}$ and $\mathrm{u}^{\prime}$ are Facebook friends

Similarity is defined as a measure of how much users' tastes are similar and is computed counting how many times the two users gave a similar rating to the same places:

$$
\operatorname{sim}\left(u, u^{\prime}, a\right)=\frac{\left\|\operatorname{Corated}\left(u, u^{\prime}, a\right)\right\|}{\left\|\operatorname{Known}(u, a) \cap \operatorname{Known}\left(u^{\prime}, a\right)\right\|}
$$

$$
\begin{aligned}
\text { Corated }\left(u, u^{\prime}, a\right)= & \bigcup\{p \in \mathcal{P} \mid \exists g \in \mathcal{G}, \\
& \text { Liked }(u, p, a, g) \wedge \operatorname{Liked}\left(u^{\prime}, p, a, g\right) \vee \\
& \text { Disliked } \left.(u, p, a, g) \wedge \operatorname{Disliked}\left(u^{\prime}, p, a, g\right)\right\}
\end{aligned}
$$

On this foundation, we study how different factors influence user preferences by analysing how effective they are in recommending places users would probably like. To this end, we define the recommendation of places $p$ to a user $u$ to perform an activity $a$ with a goal $g$ as:

1) $\operatorname{Rec}(u, a, g, k) \subseteq \operatorname{Unknown}(u, a)$,

2) $|\operatorname{Rec}(u, a, g, k)|=k$,

3) $\forall p \in \operatorname{Rec}(u, a, g, k)$

$\forall p^{\prime} \in(\operatorname{Unknown}(u, a) \backslash \operatorname{Rec}(u, a, g, k))$, $\left.(\operatorname{score}(\operatorname{Net}(u, a), p, a, g)) \geq \operatorname{score}\left(\operatorname{Net}(u, a), p^{\prime}, a, g\right)\right)$.

\section{B. The social context}

We started looking at the social context in our preliminary work [1] where we analysed the effect of the social dimension in user preferences. In this study, we collected ratings about restaurants in Trento, Italy $(\mathcal{P})$ that people recommended for eating outside $(\mathcal{A})$ considering a one-dimensional goal $(\mathcal{G})$. We gathered 694 ratings from 90 users, over a total of 75 restaurants, taken from TripAdvisor's top 75 ranked restaurants in Trento. Unlike TripAdvisor, our population was people who was living in Trento and therefore knew the restaurant 
scenes rather well. We compared the resulting ranking with those of Lonely Planet and TripAdvisor, finding no correlation in the various rankings. These initial results suggested that according to the population and purpose, these rankings could vary significantly.

Based on this, we analysed how the network of people providing the ratings affected the discovery process. Thus, we considered four different types of network and analysed the precision and recall using those networks as source of recommendations:

1) Overall population: Considers all users

$$
\operatorname{Net}_{o}(u, a)=\mathcal{U}
$$

2) Friends: Considers only friends

$$
\operatorname{Net}_{f}(u, a)=\left\{u^{\prime} \in \mathcal{U} \mid \text { FriendOf }\left(u, u^{\prime}\right)\right\}
$$

3) Similar users: Considers users with similar taste

$$
\operatorname{Net}_{s}(u, a)=\left\{u^{\prime} \in \mathcal{U} \mid \operatorname{sim}\left(u, u^{\prime}, a\right)>\delta\right\}
$$

4) Similar friends: Considers friends with similar taste

$$
\operatorname{Net}_{s f}(u)=\left\{u^{\prime} \in \mathcal{U} \mid \text { FriendOf }\left(u, u^{\prime}\right) \wedge \operatorname{sim}\left(u, u^{\prime}, a\right)>\delta\right\}
$$

With this definition of networks, we took $70 \%$ of the ratings of each user, and look at how well $\operatorname{Rec}(u, a, g)$ performed in recommending, for each network, the remaining $30 \%$.

The results suggested, not surprisingly, that recommendations coming from similar users $\left(\mathrm{Net}_{s}\right)$ performs better than overall popularity $\left(\mathrm{Net}_{o}\right)$. More interestingly, recommendations coming from friends $\left(N e t_{f}\right)$, and specially if reduced to similar friends $\left(N e t_{s f}\right)$, outperforms considerably those of other networks in both precision and recall. These results point to the importance of balancing personal tastes (in this case captured by rating behaviour) with the real social context (i.e., friends).

\section{Extending the experiment}

The study we present in this paper extends the previous one in three directions: i) the geographic area is extended to three different cities around the world; ii) in each city we asked ratings not only for restaurants but also for another activity that is usually done before or after going out for dinner; iii) for each place people were able to specify four different marks according to different goals. The experiment was run in Trento (Italy) with bars for aperitivo as second type of places, Asunción (Paraguay) with pubs, and Tomsk (Russia) with clubs. In all cases, we considered the same four purposes: one mark was dedicated to the price / quality ratio and the other three was related to the different types of companions people can spend their leisure time with, which are tourists, friends and their partner.

A website guided users through the procedure for rating the different places available for their city, requesting to access using their Facebook account (from which friends relationships are collected) and requesting at least five marked places in the first activity in order to access the list prepared for the second one. The website was published the $16^{\text {th }}$ of
November 2012 and collected people's preferences for two months. It was advertised mainly through social networks, but in Trento some posters were also hung up in the university's buildings. For Trento we asked to mark places for aperitivo first, choosing between the 30 provided, and restaurants after, with a list of 67 places. 49 people participate to the study, leaving 2700 marks. For Asunción we started with a list of 254 restaurants, followed by 43 pubs and bars, collecting a total of 6100 marks from 97 people. We started from restaurants for Tomsk too, with a list of 32 places, followed by 12 clubs. We reached 16 people that left 1020 marks. The website was designed in a way that it was very easy to filter all the available places and find the ones that are known.

\section{Understanding preferences across activities}

In the second study we focused on understanding whether it was possible to extend recommendations across different activities: knowing a user's taste for restaurants, is it possible to recommend her another activity, and a place where to perform it, using only her taste for restaurants?

Going after that question, we collected ratings for two typical activities $\left(a_{1}, a_{2}\right)$ in our three target locations. Then, for each user $(u)$, we created a dataset without all her ratings for the second activity, leaving $\operatorname{Known}\left(u, a_{2}\right)=\emptyset$. On this dataset, we calculated the recommendations assuming the following definition of network:

$$
\operatorname{Net}(u)=\left\{u^{\prime} \in \mathcal{U} \mid \exists a^{\prime} \in \mathcal{A}, \operatorname{sim}\left(u, u^{\prime}, a^{\prime}\right)>\delta\right\},
$$

which builds a set of users sharing similar tastes in $a_{1}$, since all the user ratings for $a_{2}$ were removed. In doing so, we are recommending places for $a_{2}$ (e.g., going clubbing) using the network of users with similar taste for $a_{1}$ (e.g., going out for dinner). Borrowing the previous definition of similarity, we say that user $u$ is similar to user $u^{\prime}$ if they agree in at least $70 \%$ of their ratings $(\delta>=0.7)$.

In order to understand how good these recommendations are, we computed precision for both the top ten places and the full list. Given $\mathcal{R}(u, p, g, n)$ as the set of recommendations computed for user $u$ on place $p$ for goal $g$, where $n$ is the position in the ranking, and $\mathcal{M}(u, p, g)$ as the set of marks given by user $u$ on place $p$ for goal $g$, the precision is defined as

$$
\operatorname{Precision}(u, g, n)=\frac{\|\operatorname{Good}(u, g, n)\|}{\|\operatorname{Good}(u, g, n)\|+\|\operatorname{Bad}(u, g, n)\|}
$$

where

$$
\begin{aligned}
& \operatorname{Good}(u, g, n)=\{p \in \mathcal{P} \mid R(u, p, k) \equiv M(u, p) \wedge k \leq n\} \\
& \operatorname{Bad}(u, g, n)=\{p \in \mathcal{P} \mid R(u, p, k) \neq M(u, p) \wedge k \leq n\}
\end{aligned}
$$

Recommendations were computed for all 4 different purposes, only for the users that had a similar group containing at least 3 users $(\operatorname{Net}(u)>=3$ ). The resulting precision for the top 10 recommendations and of all of them, averaged by city, are reported in table I.

As can be seen, the precision of such recommendations is high. For the places that the considered user already rated, the 
TABLE I

Precision of THE RECOMMENDATIONS

\begin{tabular}{l||l|l} 
& Precision $(10)$ & Precision $(\|\mathcal{P}\|)$ \\
\hline \hline Trento & 0.85 & 0.81 \\
\hline Asunción & 0.922 & 0.893 \\
\hline Tomsk & 0.82 & 0.84 \\
\hline
\end{tabular}

recommendations usually matched the user's rating, with very few errors that appeared mainly for users with a too wide similarity group. The results suggest that similarities in the preferences of people for an activity can be extended to other activities, which points to potential of profiling users based on lifestyle.

\section{E. Effect of goal on user preferences}

Given all the collected ratings, we first analysed the differences between the rankings resulting from the marks for different goals. All places, divided by city and by activity, were ordered, for each goal, according to the average mark and the number of marks received, building in this way four rankings for each activity in each city. We considered only the places that received at least 5 marks for each goal, maintaining only the places that collected a minimum amount of the crowd's opinion. The remaining places are 23 restaurants and 30 bars for Trento, 87 restaurants and 26 pubs for Asunción, 21 restaurants and 12 clubs in Tomsk.

Kendall $\tau$ distance was used to compute the difference between the four rankings for each activity, counting the number of couples in the rankings that appear in the same order. The returned value is between 1 and -1, with 1 meaning that the two rankings are equal, while -1 means that they are completely the opposite. Naming $C$ the number of concordant couples and $D$ the number of the discordant ones, the metric is defined with the following formula:

$$
\text { Kendall } \tau=\frac{C-D}{C+D}
$$

The Kendall $\tau$ distance is reported in tables II, III, IV, V, VI and VII, where the goals are summarised in the following way: $\mathrm{T}=$ Bringing tourists, $\mathrm{F}=$ Bringing friends, $\mathrm{P}=$ bringing the partner and $\mathrm{Q}=$ price / quality ratio.

As can be seen, in both Trento's tables II and III the highest similarity is between the rankings for Bringing tourists and Bringing the partner, while they are both different from Bringing friends and in particular from Price / quality ratio, that has almost half of the couples in the wrong order. The distance between Price / quality ratio and Bringing friends is slightly less, giving a hint that going out with friends the quality and price of food and beverages are took in consideration more than when spending time with tourists and the partner.

In Asunción, tables IV and $\mathrm{V}$, there is still a higher similarity between Bringing tourists and Bringing the partner, but their distance to Bringing friends and Price / quality ratio is much less. Moreover, both Bringing friends and Price / quality ratio are almost equally distant from all the other
TABLE II

Kendall $\tau$ DISTANCES FOR TRENTO'S RESTAURANTS

\begin{tabular}{l||l|l|l|l} 
& $\mathrm{T}$ & $\mathrm{F}$ & $\mathrm{P}$ & $\mathrm{Q}$ \\
\hline \hline $\mathrm{T}$ & 1.0 & & & \\
\hline $\mathrm{F}$ & 0.146 & 1.0 & & \\
\hline $\mathrm{P}$ & $\mathbf{0 . 6 5 2}$ & 0.162 & 1.0 & \\
\hline $\mathrm{Q}$ & -0.106 & $\mathbf{0 . 2 8 8}$ & -0.027 & 1.0 \\
\hline
\end{tabular}

TABLE III

Kendall $\tau$ DISTANCES FOR TRENTO'S BARS FOR APERITIVO

\begin{tabular}{l||l|l|l|l} 
& $\mathrm{T}$ & $\mathrm{F}$ & $\mathrm{P}$ & $\mathrm{Q}$ \\
\hline \hline $\mathrm{T}$ & 1.0 & & & \\
\hline $\mathrm{F}$ & 0.287 & 1.0 & & \\
\hline $\mathrm{P}$ & $\mathbf{0 . 6 9 6}$ & 0.232 & 1.0 & \\
\hline $\mathrm{Q}$ & -0.007 & 0.227 & 0.011 & 1.0 \\
\hline
\end{tabular}

rankings, showing that there is no stronger correlation between them. Moving to pubs and bars, we can see that the similarity between Bringing tourists and Bringing the partner is not present here, with only Bringing friends slightly more distant from the other rankings.

In Tomsk, tables VI and VII, the rankings are again divided in two groups, with Bringing tourists and Bringing the partner on one side and Bringing friends and Price / quality ratio on the other. Looking at the Kendall $\tau$ distance for club rankings, the situation is different. Here the closest rankings are Bringing friends and Bringing the partner, immediately followed by the already known couple Bringing tourists and Bringing the partner. Despite this, Bringing friends and Bringing tourists are distant.

Summarizing all the results, we have seen that people preferences are sensitive to the companion (e.g., partner, friends, tourists) for which they look for different features. In particular, most of the times going out with friends results in different choices than going out with the partner or tourists, and sometimes goes together with higher attention to the price / quality ratio. As we have seen there are some exceptions to this generalization, specially for Asunción's pubs where the price / quality ratio is considered more when choosing places where to bring tourists. These differences depending on the location can be intuitively be related to the cultural and economical aspects.

\section{DISCOVERING AND FINDING EVENTS}

Keeping in consideration the lesson learned from our studies, we designed a web interface for making it easy to find and discover events and places where people can perform social and cultural activities in their leisure time.

As can be seen in Fig. 1, at the top of the page is presented the filtering module, which helps people finding events and places:

- What: filter by type of activity that can be performed in the place,

- Where: filters by area in which the user would like to perform the activity. The results are filtered in real time as the user spans the area, 

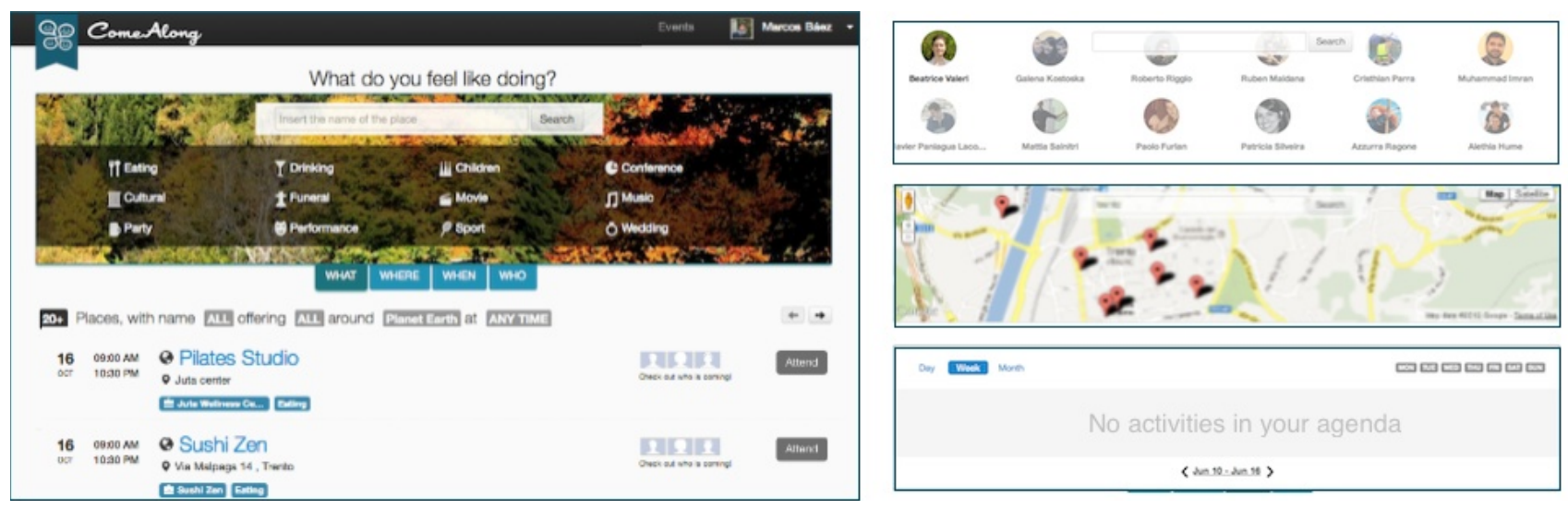

Fig. 1. ComeAlong UI, Interface for discovering events

TABLE IV

Kendall $\tau$ DISTANCES FOR ASUNCIÓN'S RESTAURANTS

\begin{tabular}{l||l|l|l|l} 
& $\mathrm{T}$ & $\mathrm{F}$ & $\mathrm{P}$ & $\mathrm{Q}$ \\
\hline \hline $\mathrm{T}$ & 1.0 & & & \\
\hline $\mathrm{F}$ & 0.383 & 1.0 & & \\
\hline $\mathrm{P}$ & $\mathbf{0 . 5 0 2}$ & 0.388 & 1.0 & \\
\hline $\mathrm{Q}$ & 0.324 & 0.328 & 0.262 & 1.0 \\
\hline
\end{tabular}

TABLE V

Kendall $\tau$ DISTANCES FOR ASUNCIÓN's PUBS AND BARS

\begin{tabular}{l||l|l|l|l} 
& $\mathrm{T}$ & $\mathrm{F}$ & $\mathrm{P}$ & $\mathrm{Q}$ \\
\hline \hline $\mathrm{T}$ & 1.0 & & & \\
\hline $\mathrm{F}$ & 0.28 & 1.0 & & \\
\hline $\mathrm{P}$ & $\mathbf{0 . 3 6}$ & 0.194 & 1.0 & \\
\hline $\mathrm{Q}$ & $\mathbf{0 . 3 3 5}$ & 0.169 & 0.261 & 1.0 \\
\hline
\end{tabular}

- When: the time range in which the user would like to perform the activity. This filter, in addition, informs the user of other activities in that range to empower her decision.

- Who: allows users to filter events and activities in which friends are participating, participated or recommended.

Each of these filters are orthogonal to each other and can be used independently or jointly. The interesting aspect of this design is that results can aggregate the different aspects to rank activities and events. Our current prototype incorporates the recommendation aspects explored in our studies.

The social aspect is deeply integrated into the platform. People can share their intention to attend an event or perform an activity and their friends can see what they plan to do. Knowing that a specific friend will participate to the event not only gives the user a good reason to participate, but it is also an extra hint on the expected quality and type of the entertainment that the specific event provides.

Users can recommend an event to their friends also through ratings, suggesting them to participate to the next occurrence of the event they just attended. Next to the event description, the average of the collected marks is shown to indicate the general "reputation" of the event, but this is not enough since
TABLE VI

Kendall $\tau$ DISTANCES FOR TOMSK'S RESTAURANTS

\begin{tabular}{l||l|l|l|l} 
& $\mathrm{T}$ & $\mathrm{F}$ & $\mathrm{P}$ & $\mathrm{Q}$ \\
\hline \hline $\mathrm{T}$ & 1.0 & & & \\
\hline $\mathrm{F}$ & 0.019 & 1.0 & & \\
\hline $\mathrm{P}$ & $\mathbf{0 . 4 7 6}$ & -0.067 & 1.0 & \\
\hline $\mathrm{Q}$ & -0.133 & $\mathbf{0 . 4 2 9}$ & -0.029 & 1.0 \\
\hline
\end{tabular}

TABLE VII

Kendall $\tau$ DISTANCES FOR TOMSK'S CLUBS

\begin{tabular}{l||l|l|l|l} 
& $\mathrm{T}$ & $\mathrm{F}$ & $\mathrm{P}$ & $\mathrm{Q}$ \\
\hline $\mathrm{T}$ & 1.0 & & & \\
\hline $\mathrm{F}$ & 0.182 & 1.0 & & \\
\hline $\mathrm{P}$ & $\mathbf{0 . 4 5 4}$ & $\mathbf{0 . 5 4 5}$ & 1.0 & \\
\hline $\mathrm{Q}$ & 0.0 & 0.273 & 0.242 & 1.0 \\
\hline
\end{tabular}

personal tastes can be different from the overall population. For this reason, we decided to show the single ratings given by user's friends. Knowing their tastes and how different they are from hers, the user can better understand how much she could like the event. In this way, the user gets extra information thanks to her social network and have more possibilities to choose an event that she will really enjoy.

\section{Motivating USER PARTICIPATION}

With a better understanding of the dynamics of users preferences, we moved our focus on how to motivate people to participate to events. This change in the perspective allowed us to understand how event organisers and place owners can attract more people to their events and places. In the following we present a study done with a famous Italian museum and present the resulting design of an application to motivate visitors to attend museum activities.

\section{A. Why do people come to the museum?}

Following this question we run a study in collaboration with the MART ${ }^{11}$ museum, starting with a short structured inter-

\footnotetext{
${ }^{11}$ Museum of contemporary art of Rovereto, Trento, http://www.mart.trento.it/
} 


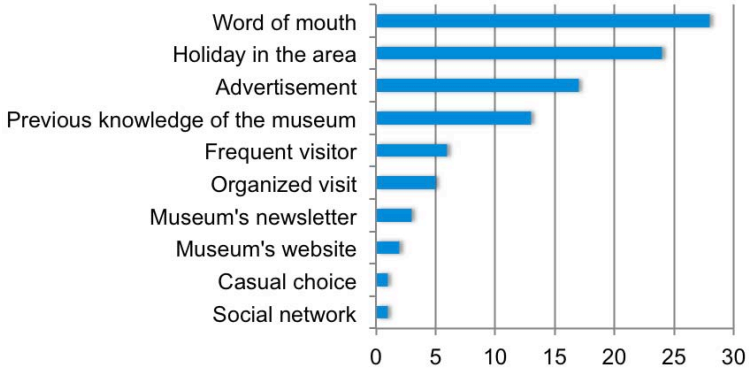

Fig. 2. What triggered your decision to come to the museum?

view. During the $23^{\text {rd }}$ and $24^{\text {th }}$ March 2013, we interviewed 208 people at the end of their visit, which correspond to the $23 \%$ of the visitors in that weekend. This first part was focused on the decision to come to the museum: when they decided to come and why. Surprisingly, more than half of the respondents decide to go to the museum the same day or the one before, suggesting contextual factors influencing an immediate decision. The biggest part of the rest of respondents, instead, plan the visit in advance, at least two weeks before. People mainly visit the museum because of their personal interests (e.g., curiosity and for relaxing, for a total of $81 \%$ ), but still there is a $9 \%$ of visitors that come along with their family and friends just to engage in a social activity. This is an evidence of strength of the social component in this particular scenario.

Digging deeply into what specifically triggered their decision to visit this museum, we found out that word of mouth is what motivates people to come the most, closely followed by a contextual decision: being in the city for holidays (Figure 2). Grouping together all the other items that depend on the museum's marketing and image, they cover $41 \%$ of the respondents, maintaining their first place in triggering people's interest. What is more significant for us is the low actual impact of social networks on the decision to come at the museum. This indicates that there is space for improvements through this communication channel.

In order to understand better whether and how people recommend to visit the museum, we sent to the interviewed people a follow-up questionnaire by email the next Wednesday and we received 62 answers (the $30 \%$ of the sent emails). In the results, most visitors recommended to their family and friends to go to the museum, but only $5 \%$ of them did it also through social networks. This suggests that, even though the museum is present on the most used social networks, very few people share their museum experiences through this channel. This result supports our idea that museums are not exploiting the full potential of social networks, and our goal is to increase the rumors about museums by motivating word of mouth through it.

\section{B. ComeAlong for museums}

ComeAlong for museums was designed with the goal of attracting more people to museum activities by i) providing a channel for communicating those activities, ii) motivating people to share their intention to participate, and iii) exploiting social ties to bring more people to the museum.

On this premise, the application provides users with a direct channel for the museum communications. Together with the information about museum events and exhibitions, it also provides announcements, keeping users always informed of everything that concerns the museum. It is designed to attract initially people that are already interested in museums, creating a personal and stronger relationship with them.

To increase the intention sharing on social networks, ComeAlong facilitates and encourages sharing by i) integrating the platform with Facebook, requiring only one click to share, and ii) providing incentives (defined by the museum) for people to share their intention to come to the museum, which translates into a native advertisement message ${ }^{12}$ published on the users' social network, inviting their friends and contacts to come along.

The benefit offered by the museum can vary from a gadget of the museum, which reminds users of their visits, to a discount on the ticket or also a free entry. The decision of the benefit is important since it has to be interesting enough for motivating people to give up their privacy, but also cheap enough to compensate its costs with the extra people coming at the museum thanks to the word of mouth resulting form the usage of the platform. Balancing this trade-off is an interesting aspect to explore.

ComeAlong for museums will be tested at the MART museum for motivating people to visit its exhibitions and the events related to them. Different benefits will be tested in the events organized throughout the summer, giving us the opportunity to understand people's reaction to them. The application will be used also for advertising the opening of the $\mathrm{MuSe}^{13}$, the new museum of sciences of Trento, on the $27^{\text {th }}$ July 2013.

\section{ARCHITECTURE}

The ComeAlong platform, as seen in Figure 3, relies on a service-oriented architecture, with a set of core modules at the bottom exposing a RESTful API to the vertical applications on the top. While the platform is able to manage all kind of events, the vertical application can specialize the interface to the context and the needs of the vertical domains.

The services of the platform are built around four main modules:

- Activity management is the module that manages the creation and retrieval of activities, places, ratings, and all the information that requires starting and running an activity.

- Discovery module provides all services to help people find the interesting events and activities. It contains all the functions for filtering and recommending events.

- Statistics provides useful data on the participation to activities and usage of the application, such as people that

\footnotetext{
${ }^{12} \mathrm{http} / / /$ en.wikipedia.org/wiki/Native_advertising

${ }^{13}$ http://www.muse.it/
} 


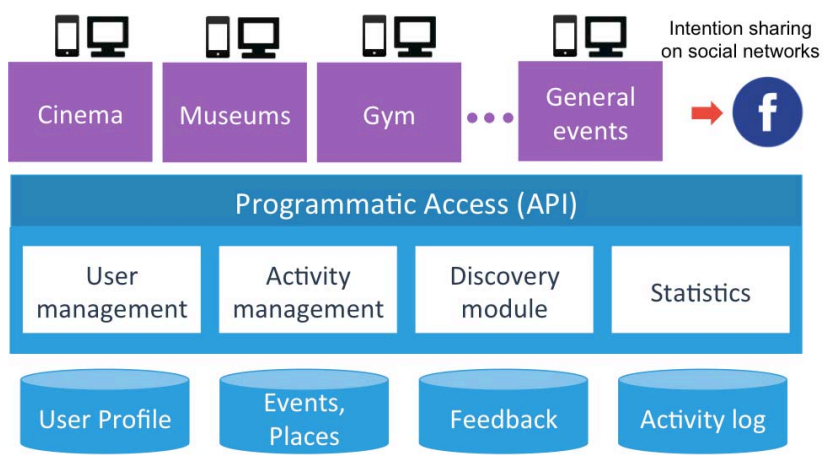

Fig. 3. ComeAlong architecture

share their intention, aggregated feedback on activities, influential users, etc.

- User management manages user authentication and user personal data.

The actual intention sharing is left to the vertical application, which can easily publish a message on the user's social network thanks to the provided API. This design decision provides more flexibility to vertical application to control what, how and when things are being shared.

The ComeAlong platforms is very generic and can manage any type of leisure events and activities, but it can be used also to build context-specific web and smartphone applications. These applications can provide specific user interface and services for museums, as we have already seen, but also different contexts such as movie theatres and gyms. We already developed a prototype application for movie theatres, with the specific use case of Cinema Astra in Trento. In this specific case, users are supported in the choice of the movie show to attend, with the possibility to book their ticket in advance, obtaining a discount too. The effects of using the application have not been tested yet, but its implementation is a prove that the general-purpose platform can be used in many different contexts, moving from culture to leisure without any problem. Moreover, we're currently in the process of designing a version of ComeAlong for a totally different context: a gym. Even in this case, the platform, thanks to its generality, is able to provide the needed support for the context-specific UI.

\section{DISCUSSION AND FUTURE WORK}

In this paper we have studied various aspects around user preferences that could be used to help users find activities and places they would actually like. On a different perspective, we have also seen how event organisers could attract more people to their events.

Through the studies about recommendations, we found that the knowledge about the users' preferences and neighbours on one specific activity (i.e., restaurants) can be used to recommend another related activity (i.e., a common saturday evening activity) even without any information about a specific user preferences on the second activity, reducing the cold start problem of collaborative filtering. Moreover, thanks to this we can provide better recommendations, extending the knowledge-base to different activities.

In the museum context, we focused on the understanding of the current triggers that motivate people to go to the museum and we found that word of mouth is very important, but it does not propagate through social networks, remaining limited mainly to face-to-face interactions. This is an important finding, showing that there is a possibility to improve word of mouth and make more people aware of the cultural events.

Based on our initial findings we have designed and built prototypes for discovering events and motivating people to participate to events. In the upcoming months we will be testing these prototypes in collaboration with two museums important museums in Italy: MART and MuSe. With these pilots we are planning to study the actual effect of our designs in helping the participation to events.

\section{REFERENCES}

[1] B. Valeri, M. Báez, and F. Casati, "Comealong: Empowering experiencesharing through social networks." in CollaborateCom. IEEE, 2012.

[2] E. M. Daly and W. Geyer, "Effective event discovery: using location and social information for scoping event recommendations," in RecSys, 2011.

[3] J. Miguéns, R. Baggio, and C. Costa, "Social media and tourism destinations: Tripadvisor case study," Advances in Tourism Research, 2008.

[4] I. Jeacle and C. Carter, "In tripadvisor we trust: Rankings, calculative regimes and abstract systems," Accounting, Organizations and Society, 2011.

[5] P. O' Connor, "User-generated content and travel: A case study on tripadvisor. com," Information and communication technologies in tourism, 2008.

[6] H. Cramer, M. Rost, and L. Holmquist, "Performing a check-in: emerging practices, norms and'conflicts' in location-sharing using foursquare," in Mobile HCI, 2011.

[7] J. Lindqvist, J. Cranshaw, J. Wiese, J. Hong, and J. Zimmerman, "I'm the mayor of my house: examining why people use foursquare-a socialdriven location sharing application," in CHI 2011, 2011.

[8] L. Backstrom, E. Sun, and C. Marlow, "Find me if you can: improving geographical prediction with social and spatial proximity," in $W W W ' 10$, 2010.

[9] J. Chang and E. Sun, "Location3: How users share and respond to location-based data on social networking sites," in ICWSM-11, 2011.

[10] A. Noulas, S. Scellato, C. Mascolo, and M. Pontil, "An empirical study of geographic user activity patterns in foursquare," in ICWSM-11, 2011.

[11] L. Mui, P. Szolovits, and C. Ang, "Collaborative sanctioning: applications in restaurant recommendations based on reputation," in AGENTS '01, 2001.

[12] P. Bonhard, C. Harries, J. McCarthy, and M. A. Sasse, "Accounting for taste: using profile similarity to improve recommender systems," in $\mathrm{CHI}$ '06, 2006.

[13] R. Schenkel, T. Crecelius, M. Kacimi, T. Neumann, J. X. Parreira, M. Spaniol, and G. Weikum, "Social wisdom for search and recommendation." IEEE Data Eng. Bull., 2008.

[14] F. Liu and H. J. Lee, "Use of social network information to enhance collaborative filtering performance," Expert Systems with Applications, 2010.

[15] H. Ma, D. Zhou, C. Liu, M. R. Lyu, and I. King, "Recommender systems with social regularization," in WSDM '11, 2011.

[16] M. Kayaalp, T. Ozyer, and S. Ozyer, "A collaborative and content based event recommendation system integrated with data collection scrapers and services at a social networking site," in ASONAM '09, 2009.

[17] B. Li, Q. Yang, and X. Xue, "Can movies and books collaborate?: crossdomain collaborative filtering for sparsity reduction," in IJCAI'09, 2009. 